\title{
Advancement in Pain Relief Neuro Surgical Techniques for Pain Relief
}

\author{
Ashfaq Ul Hassan ${ }^{1, *}$, Villayat Buch ${ }^{2}$, Pervez Shah ${ }^{3}$ \\ ${ }^{1}$ MBBS, MS , Lecturer SKIMS Medical College Bemina, India \\ ${ }^{2}$ MBBS, MD Assistant Professor Anesthesiology, SKIMS, Soura, India \\ ${ }^{3}$ MD, DM Neurology, SMHS Hospital Srinagar, India \\ *Corresponding Author: ashhassan@rediffmail.com
}

Copyright (C) 2013 Horizon Research Publishing All rights reserved

\begin{abstract}
The most common symptom leading to a doctors clinic is pain and Pain is the most common symptom that makes any patients to seek medical advice and assistance. Grading pain and failure to respond to conventinal and non convential drugs leads to Neurosurgical intervention for the relief of pain which is indicated when primary intervention for the disease in question does not lead to adequate comfort. Surgical procedures to reduce pain are grouped broadly into two categories: neuroablative and neuroaugmentative. Taking account of the Neuroanatomy, Ablative procedures can be done anywhere along this pathway. Neuroaugmentation refers to the use of chronic infusion of opioid agonists or stimulation, which is believed to activate intrinsic modulating systems that reduce or alter sensory input.
\end{abstract}

Keywords Neursurgery, Pain, Ablative, Spinal Cord, Rhizotomy, Tractotomy, Thalomotomy

\section{Introduction}

The most common symptom leading to a doctors clinic is pain and Pain is the most common symptom that makes any patients to seek medical advice and assistance.

Grading pain and failure to respond toconventional and non convential drugs leads to Neurosurgical intervention for the relief of pain which is indicated when primary intervention for the disease in question does not lead to adequate comfort.

Clinically, pain can be classified temporally as acute or chronic; quantitatively as mild, moderate, or severe; physiologically as somatic, visceral, or neuropathic; and etiologically as medical or psychogenic. Patients with acute pain usually give a clear description of its location, character, and timing, leading to an etiologic diagnosis. Objective signs and associated autonomic nervous system hyperactivity with tachycardia, hypertension, and diaphoresis are present. Multidisciplinary approaches to treatment play a critical role in addressing the multidimensional aspects of the pain. Other commonly used terms to describe pain include baseline pain, which refers to the average pain intensity expressed for 12 or more hours in a 24-hour period, and breakthrough pain, which is a transient increase in pain resulting from volitional (e.g., incident pain on movement) and nonvolitional factors (flatulence).

Surgical procedures to reduce pain are grouped broadly into two categories: neuroablative and neuroaugmentative. Pharmacologically distinct receptors located in the periphery code for various stimuli. The sensory experience is, however, a summation of the specific receptor activation and the modulation applied by the central nervous system to that resulting input. In general, nociceptors consist of free nerve endings, and painful sensations are transmitted in small, unmyelinated A delta and $\mathrm{C}$ nerve fibers. In Addition there are GABA receptors, Substance $P$ and Peptidergic receptors present in the Ganglion. Cell bodies are located in the dorsal root ganglia, and axons enter the spinal cord through the dorsal roots. There is convergence of pain fibers more laterally in the root, and Lissauer's tract separates the dorsal horn from the cord surface. These axons synapse with second-order neurons in the dorsal gray of the cord. The majority of pain and temperature information is transmitted in the lateral spinothalamic tract to the thalamus, where third-order neurons are located.

In the Present manuscript we are going to summarize the various techniques required for relief of unrelenting pains not controlled ordinarily by conventional pharmacological agents.

\section{Neuro Ablative Techniques}

\subsection{Neurectomy}

Despite the fact that imagining the fact that simply destruction of a nerve would cause relief of pain, there is little role for the transection of nerves to relieve pain. Cutting or dividing a nerve called Neurectomy is not routinely used 
for simple pains but can be used selectively to treat painful neuromas. A number of procedures have therefore been described to prevent this recurrence, such as

- burying the nerve in bone

- burying the nerve in muscle

- or covering the nerve with Silastic

- separating the two nerve ends

The technique is not fully effective as Most peripheral nerves are mixed, reducing the value of transection unless motor loss is acceptable. A notable exception is the superficial branch of the dorsal interosseous nerve in the forearm, which is best treated by transection rather than attempted repair following injury. It is a purely sensory nerve. Avulsion or alcohol ablation of the infraorbital, supraorbital, or mental nerve has a valuable role in the treatment of trigeminal neuralgia, although the benefit is often temporary. Denervation of the facet joints in the spine is sometimes attempted to relieve chronic low back pain, but this therapy is controversial. [1]

\subsection{Rhizotomy}

The technique refers to ablation of the sensory root. This can be done either as an open procedure, intradurally or extradurally, [22] or as a percutaneous procedure using radiofrequency coagulation [34] or injection with phenol. The most common indication is for rhizotomy pain following unsuccessful disc procedures. Leg symptoms are prominent and are presumably related to permanent root irritation. The results are not encouraging. Rhizotomy is useful for pain secondary to tumors involving a limited dermatomal region. It has been used with limited success in postherpetic neuralgia and postthoracotomy pain. Sometimes this causes discomfort at the denervation margin, producing a growing area of abnormal sensation and associated pain.

Most Patients selected for rhizotomy undergo a preoperative series of selective root blocks. Subsequent pain relief does not guarantee equally successful surgical outcome. There is considerable variability in the dermatomal distribution, with much overlap, and some sensory fibers travel in the ventral root. Therefore, several surgeons have turned to ganglionectomy to eliminate all sensory fibers at a specific level.[19] To produce any long-term sensory loss, at least three roots must be taken. Sacrifice of more than two sensory roots in an extremity can lead to loss of function, in spite of preserved motor capacity.

Technically the procedure is simple, but accurate localization is tough. Stimulation in the lumbar region is usually used, since with the intra-arachnoid approach, the roots are identified near the conus at some distance from their exiting foramina. The roots or ganglia can be identified at the exiting foramen in an extra-arachnoid fashion, which is often easier. Care is taken to avoid vascular injury, as the blood supply to the cord enters along the roots.

\subsection{Retrogasserian Rhizotomy for Trigeminal Neuralgia}

Pain in the face that involves the distribution of the lower cranial nerves should be considered a distinct entity, and it responds to denervation more favorably. Trigeminal neuralgia consists of intermittent bouts of lancinating pain that is often triggered and is limited to the trigeminal distribution in a patient who is neurologically intact. Most patients are over 55 years of age. It is basically A disorder of the trigeminal nerve producing bouts of severe, lancinating pain lasting seconds to minutes in the distribution of one or more of its sensory divisions, most often the mandibular and/or maxillary. Adults usually are affected, especially later in life. The pain is often set off by touching a trigger point or by activity (eg, chewing or brushing the teeth). Although each bout of intense pain is brief, successive bouts may incapacitate the patient Unsuccessful treatment with Carbamazepine, Phenytoin, baclofen, amitriptyline or trazodone leads to think about surgical intervention.

Retrogasserian ganglion compression,[6]glycerol ablation,[7] and radiofrequency lesioning [5] are variations of rhizotomy. All three are performed percutaneously, with fluoroscopic control, while the patient is under sedation and they can incorporate specific localization by stimulation. In an elderly patient with idiopathic trigeminal neuralgia, these procedures represent the treatment of choice when medical management with carbamazepine, phenytoin, or baclofen has failed. In a younger patient, or one who considers sensory loss unacceptable, microvascular decompression of the trigeminal root entry zone is preferred,[ 8 ] but this has the added risk of open craniectomy. At times, vascular decompression is combined with open partial caudal root section.[38]

\subsection{Dorsal Root Entry Zone Lesioning}

Lesioning of the dorsal root entry zone (DREZ) with a radiofrequency current, using a $0.25-\mathrm{mm}$. diameter electrode with a $2.0-\mathrm{mm}$. exposed tip, was introduced by Nashold and Ostdahl in 1979.[ 18 ]Lissauer's tract and the dorsal horn gray matter are coagulated down to the Rexed V lamina, including the second-order neurons in the sensory and pain pathway. Lesions are made in line, close enough together so as to coalesce and produce a continuous zone of obliteration. The greatest success is achieved for pain secondary to plexus avulsion and spinal cord injury. Intermittent spontaneous or induced pain, usually in a transition band located approximately at the level of the spinal cord injury, responds most consistently.

DREZ lesions have also been made with lasers. Sindou and co-workers introduced a technique in which a longitudinal incision is made into the cord at the lateral edge of the sensory root. This procedure was used mostly for spasms, but the authors report equal success in the treatment of pain.[ 26, 27]

\subsection{Caudalis DREZ Coagulation}

Nucleus caudalis DREZ coagulation was introduced as an 
extension of DREZ lesioning in 1982.[ 3] The trigeminal nucleus caudalis contains second-order neurons subserving pain, temperature, and crude touch from the fifth, seventh, ninth, and tenth cranial nerves.

This procedure has been performed for anesthesia dolorosa secondary to multiple procedures for trigeminal neuralgia ,postherpetic neuralgia , atypical facial pain , pain following facial or dental trauma, central pain following stroke, cluster headache , multiple sclerosis , trigeminal tumor, and brain stem injury following stereotactic radiation.

\subsection{Cordotomy}

Edinger first described the spinothalamic tract in 1889 , and in 1905, Spiller observed dissociated contralateral sensory loss in a patient with a tuberculoma, which compressed the anterior quadrant of the spinal cord. In 1912, Spiller was able to convince Martin to perform an open cordotomy in a patient with rectal carcinoma and intractable pain, with successful pain relief.[ 30]Mullan's[ 16 ]innovation led to the current technique of high percutaneous radiofrequency cordotomy. [13 ]This procedure is done with $\mathrm{x}$-ray guidance in an awake patient and is therefore well tolerated, even by those who are very sick with advanced disease. The goal is to coagulate the spinothalamic tract in the anterior cord and ventral to the dentate ligament, which can be visualized myelographically. Impedance is used to identify penetration of the cord. Stimulation confirms localization within the cord when the patient describes hot, cold, or paresthesia on the contralateral side, in the absence of ipsilateral motor responses.

Cordotomy is most useful in patients with cancer who have unilateral pain in the trunk or lower extremity; however, it is possible to treat upper extremity pain. Bilateral procedures should be separated by at least 2 weeks. Immediate pain relief is excellent .[13 ]There is loss of pain relief with time, and the procedure has not proved to be effective in benign conditions such as failed back, shingles, or causalgia. Complications include ipsilateral leg weakness, bladder dysfunction, respiratory alteration, and anesthesia dolorosa. Ondine's curse occurs almost exclusively following bilateral procedures and consists of a loss of involuntary respiratory drive, causing apnea and possible death. It is usually transient. Failure to respond to a $\mathrm{CO} 2$ challenge with hyperventilation may predict this complication preoperatively. Some patients describe severe ipsilateral pain after their original contralateral pain is successfully eliminated. Open cordotomy is largely of historical interest.

\subsection{Myelotomy}

Myelotomy, first performed in 1927 by Armour, 1 also takes advantage of the crossed nature of the pain pathway. It consists of splitting the spinal cord in a midline sagittal plane, usually at and above the level of pain. Myelotomy is of particular value for bilateral and midline pain, especially pain involving the perineum. The resulting pain relief is widespread and often extends beyond the area of analgesia. A series of cervical myelotomies has been described for relief of pain in various locations, including the rectum.[ 21 ]Myelotomy has not gained popularity due to the extensive nature of the procedure and the risk of producing dorsal column dysfunction. Injury to the anterior spinal artery must also be avoided. This ablative procedure is effective for nociceptive pain, and its use is largely limited to pain secondary to malignancy.

\subsection{Midbrain Tractotomy}

Midbrain tractotomy consists of stereotactic ablation of the spinothalamic tract at the level of the midbrain, just below the superior colliculus. At this level, the tracts from the face and body are close to each other, with the face represented more medially.

Tractotomy has been performed throughout the brain stem, including the pons and the medulla. The midbrain lesion is positioned so as to include part of the adjacent periaqueductal gray, which constitutes the paleospinothalamic pathway. This is believed to cause a greater reduction in the emotional suffering associated with chronic pain.[25] Midbrain tractotomy is most commonly indicated for head and neck cancer, with pain involving the face and shoulder region that cannot be successfully treated by cordotomy. The most common complication is diplopia and loss of upward gaze. Diplopia usually resolves within 3 to 8 weeks and is much less common following lesions that are made $5 \mathrm{~mm}$. lower than originally described by Spiegel and Wycis.[ 29, 36 .]

\subsection{Thalamotomy}

Along with the introduction of human stereotactic surgery, Spiegel and Wycis are credited with initiating thalamotomy for the management of pain. [29] In spite of the fact that the ventrocaudal nucleus of the thalamus constitutes the termination of the lateral spinothalamic tract, thalamotomy has not achieved widespread use. The most commonly selected target is in the medial thalamus, which consists of the intralaminar nucleus, parafascicular nucleus, and centromedian nucleus. These nuclei receive input from the brain stem reticular formation. Such lesions produce widespread pain relief without demonstrable analgesia and are indicated for pain secondary to cancer that is not amenable to procedures such as cordotomy.

An alternative target site involves the posteromedial thalamus and pulvinar, with lesions at this site producing no motor or sensory deficit. Little is known about pulvinar physiology. A fourth variation of thalamotomy consists of lesions made in the dorsomedial and anterior thalamic nuclei, which project to the frontal and limbic lobes. These lesions reduce suffering but do not produce detectable changes in the ability to appreciate noxious stimuli, a situation similar to 
cingulotomy.

Thalamotomy is performed stereotactically with the patient awake. Computed tomography (CT) is most commonly used for imaging,[ 33 ] but MRI is gradually becoming more useful, since the brain stem structures can be defined. Ventriculography has largely been replaced. Lesions are typically made with radiofrequency current, although in some series, gamma knife radiosurgery was employed. Electrophysiologic confirmation of the target site is believed to be essential. Comparing reported series is difficult because of varying pain etiologies and the frequent use of multiple lesions.[ 32]

Destructive lesions of the thalamus are probably useful in relieving diffuse pain secondary to cancer or the intermittent neuralgic pain or allodynia and hyperpathia present in some patients with neural injury pain. In general, however, stimulation is more beneficial for neural injury pain.

Hypophysectomy should be mentioned, although it is rarely performed. The mechanism for effectiveness of this procedure is unknown; it was introduced to treat hormone-dependent cancer and its pain. Levin [14 ] suggests high succes rate for pain relief, with benefit even in non-hormone-sensitive malignancies.

\subsection{Cingulotomy}

Cingulotomy consists of lesioning the white matter deep to the cingulate gyrus, which represents the frontal lobe component of Papez's limbic lobe. An alternative procedure that is still actively performed, but less commonly for pain, consists of subcaudatecapsulotomy, or lesioning the inferior medial frontal lobe.

Reports suggest that pain secondary to cancer is relieved in most of the patients following cingulotomy or cingulotomy combined with midbrain tractotomy but cingulotomy attenuates withdrawal symptoms in both animals and humans.

The procedure is performed stereotactically, with radiofrequency lesions targeted 2 to $4 \mathrm{~cm}$. posterior to the tip of the frontal horn, $1 \mathrm{~mm}$. above the ventricle, and $1.3 \mathrm{~cm}$. lateral to midline; it is done bilaterally. Complications are relatively few; in fact, neuropsychologic testing suggests few changes and even an increase in IQ. Transient headache and fever, as well as transient incontinence, are common. Postoperative hemorrhage can be fatal but is rare, and seizures occur in a small proportion of patients.

\subsection{Sympathectomy}

Sympathectomy is a unique form of neuroablation that is indicated for the treatment of causalgia, reflex sympathetic dystrophy, or Raynaud's phenomenon; it is also used to relieve visceral pain, since afferent fibers from the viscera travel in the sympathetic nervous system. The explanations for pain relief following sympathectomy include the fact that afferent fibers to the extremities are present in the sympathetics, that abnormal efferent activity in sympathetic nerves maintains pain. Reflex sympathetic dystrophy follows amazingly minor injuries. Left untreated for prolonged periods, both conditions progress through three phases that eventually lead to permanent conditions, with osteoporosis and trophic changes in skin and deeper tissues. If sympathetically mediated pain is treated early, usually by systemic sympathetic blockers such as phenoxybenzamine or a series of repeated sympathetic blocks, Bonica estimates an $80 \%$ cure rate. [4]Hannington-Kiff introduced regional sympathetic block using Bier's block and guanethidine to produce chemical sympathectomy,[ 11 .]

For upper extremity pain, several approaches for sympathectomy of $\mathrm{T} 2$ and $\mathrm{T} 3$ are available, including supraclavicular, transaxillary, or posterior costotransversectomy. Limiting the resection to the lower stellate ganglion limits the incidence of Horner's syndrome (ptosis, miosis, anhidrosis, and apparent enophthalmos). [12] However, complete denervation may require section up to the T11 ganglion. Risks include regeneration, pain recurrence, pneumothorax, postsympathectomy neuralgia, and impotence following bilateral lumbar sympathectomy. Celiac plexus ablation, usually done percutaneously, is very effective for the pain of pancreatic cancer or pancreatitis.

\section{The Neural Augmentation Techniques}

\subsection{Spinal Cord Stimulation}

Based on the gate control theory,[ 15] spinal cord stimulation (SCS) was first attempted in 1966.[24] The main concept involves the complex interplay between noxious and nonnoxious input in the spinal cord, which influences the ascending activity signaling pain.

SCS is generally indicated for neuropathic as opposed to nociceptive pain. Transcutaneous electrical nerve stimulation (TENS) was introduced as a method for screening patients; currently, patients are screened with a percutaneously placed temporary test electrode. The majority of late failures occur within the first year; thereafter, the results are fairly stable.[ 20 ]Stimulation is used for

- arachnoiditis,

- deafferentationrhizopathy

- multiple sclerosis

- peripheral nerve injuries

- peripheral vascular disease.

- postamputation pain

- postherpetic neuralgia

- reflex sympathetic dystrophy

- spinal cord lesion,

Stimulation is generally not effective for brachial plexus avulsion but is used for other plexus lesions. The most common indication is for the failed back syndrome or arachnoiditis, with leg symptoms responding better than back pain.[20 ]Paresthesia must be produced in the entire painful region. The electrode is placed in the mid to lower thoracic region for lower extremity pain and in the mid 
cervical region for upper extremity pain.

Complications are generally minor, and the most common is loss of effectiveness. Equipment malfunction can necessitate replacement. Some surgeons prefer to place larger electrodes through a small laminectomy in the hope of reducing migration. Infection occurs in about $5 \%$, and injury to the spinal cord is rare, as is hematoma.

PNS uses the same concept but has the advantage of being more specifically applied directly to an individual nerve. Such specific injuries are relatively uncommon, however. There is usually sufficient differential threshold between motor and sensory effect.

\subsection{Deep Brain Stimulation}

This is a technique performed since the mid-1970s. the mechanism is Stimulation of the periaqueductal or periventricular gray (PVG) is believed to be effective by means of the endogenous opioid mechanism and is most effective for nociceptive pain. It is most commonly employed for failed back syndrome and is reversible with naloxone. [39 ]The other common target is the sensory thalamus, which is most effective for neuropathic pain. Its mechanism of action is believed to be based on the gate control theory of pain modulation. Indications include

- anesthesia dolorosa

- central pain,

- cord injury

- peripheral denervation.

- postherpetic neuralgia

Infection hemorrhage or stroke are rare. In many ways, this procedure can be considered reversible - a substantial advantage in these conditions, which are difficult to treat.

\subsection{Intraspinal Narcotic Analgesia}

This technique has affected pain management to the same extent that long-acting morphine altered the care of patients with terminal cancer. Consequently, neuroablative procedures are being done less frequently. Narcotics act directly on opioid receptors in the substantiagelatinosa of the DREZ of the spinal cord. [28] The potent analgesic effect of intrathecal morphine was reported with success as early as 1976,[37] and results from human studies demonstrating the effectiveness of intrathecal[35 ]and epidural[ 2] morphine were reported shortly thereafter.

Following intrathecal injection, it has been seen that the serum levels of morphine are negligible. The onset of action is 4 to 10 minutes, and the effect lasts 11 to 30 hours. Epidural drug administration leads to a slower onset of analgesia and exposes the drug to greater redistribution into the epidural vasculature; this causes higher serum levels but limits cerebrospinal fluid redistribution, producing a more regionalized effect.

Preservative-free morphine can be delivered into the intrathecal or epidural space by continuous infusion using an implanted programmable pump. Quality of life is improved, and many patients are able to reduce or eliminate the intake of oral or parenteral narcotics. [9] Dosage requirements vary among individuals, but there is a trend to double or triple the dose over the life span of the patient. Early dose requirements for intrathecal morphine are about $5 \mathrm{mg}$. per day, and patients have been maintained for up to 3 years. This technique is indicated in those who have narcotic-responsive pain that is not adequately controlled, usually due to side effects, by systemic opioids. Continuous intraspinal infusion usually causes more potent analgesia with fewer unpleasant effects. A test dose is employed prior to implantation to demonstrate pain reduction of at least $50 \%$ sustained for 8 hours. The use of this technique in benign pain is controversial.

Complications are Catheter occlusion, Drug-related complications consist of pruritis, nausea, urinary retention, dependent edema, and respiratory depression, respiratory depression has been surprisingly rare. Nonnarcotic agents that influence neural modulation, such as low-dose intraspinal bupivacaine, alpha agonists such as clonidine, drugs that increase serotonin such as fenfluramine, gamma-aminobutyric acid (GABA) agonists, somatostatin agonists, N-methyl-D-aspartate (NMDA) receptor antagonists, or calcium channel blockers have a role.

\section{Summary}

Although these procedures are never the first line procedures for management of pain, neither are they indicated in simple pain. But sometimes intractable pain can be managed quite effectively by these procedures based on profound knowledge of Neuroanatomy. The decision to treat chronic pain by surgical means is a major decision taken by experts and sometimes difficult one, quite apart from choosing the appropriate procedure. The immense pain of cancer or any disease condition can be effectively managed by progressive use of analgesics, adjuvant pharmacologic agents, and narcotics followed by intraspinal narcotic analgesia.

\section{REFERENCES}

[1] Armour, D.: Surgery of spinal cord and its membranes. Lancet, 1:691, 1927.

[2] Behar, M., Olshwang, D., and Magora, F.: Epidural morphine in treatment of pain. Lancet, 1:527, 1979.

[3] E. J., Nashold, B. S., Jr., Caputi, F., and Moossy, J. J.: Nucleus caudalis DREZ lesions for facial pain. Br. J. Neurosurg., 1:81, 1987.

[4] Bonica, J. J.: Causalgia and other reflex sympathetic dystrophies. In Liebeskind, J. C., and Albe-Fessard, D. G. (Eds.): Advances in Pain Research and Therapy, Vol. 3. New York, Raven Press, 1979, p. 141. 
[5] A., Lazio, G., Giorgi, C., and Servello, D.: Long-term results of percutaneous retrogasserianthermorhizotomy for "essential" trigeminal neuralgia: Consideration in 1000 consecutive patients. Neurosurgery, 26:783, 1990.

[6] McDaniel, M. D., and Weaver, M. T.: Percutaneous trigeminal compression for treatment of trigeminal neuralgia: Results in 50 patients. Neurosurgery, 32:570, 1993.

[7] Burchiel, K.: Percutaneous retrogasserian glycerol rhizolysis in the management of trigeminal neuralgia. J. Neurosurg., 69:361, 1988.

[8] Clarke, H., Haglund, M., and Loeser, J. D.: Long-term efficacy of microvascular decompression in trigeminal neuralgia. J. Neurosurg., 69:35, 1988.

[9] Gorecki, J. P.: Intrathecal narcotic analgesia. In Wilkins, R. H., and Rengachary, S. S. (Eds.): Neurosurgery. New York, McGraw-Hill, 1995.

[10] Gorecki, J. P., and Nashold, B. S., Jr.: The Duke experience with the nucleus caudalis DREZ operation. ActaNeurochir., 1995.

[11] Hannington-Kiff, J. G.: Intravenous regional sympathetic block with guanethidine. Lancet, 1:1019, 1974.

[12] Kux, M.: Thoracic endoscopic sympathectomy for treatment of upper-limb hyperhidrosis. Lancet, 1:1320, 1977.

[13] Lahuerta, J., Bowsher, D., Lipton, S., and Buxton, P. H.: Percutaneous cervical cordotomy: A review of 181 operations on 146 patients with a study on the location of "pain fibers" in the C-2 spinal cord segment of 29 cases. J. Neurosurg., 80:975, 1994

[14] Levin, A. B.: Stereotactic chemical hypophysectomy. In Lunsford, L. D. (Ed.): Modern Stereotactic Neurosurgery. Boston, MartinusNijhoff, 1988, p. 365.

[15] Wall, P. D.: Pain mechanisms: A new theory. Science, 150:971, 1965.

[16] Mullan, S., Harper, P. V., Hekmatpanah, J., Torres, H., and Dobbin, G.: Percutaneous interruption of spinal-pain tracts by means of a strontium-90 needle. J. Neurosurg., 20:931, 1963.

[17] Nashold, B. S., Jr., El-Naggar, A. O., Ovelmen-Levitt, J., and Muwaffak, A.: A new design of radiofrequency lesion electrodes for use in the caudalis nucleus DREZ operation. J. Neurosurg., 80:1116, 1994.

[18] Nashold, B. S., Jr., and Ostdahl, R. H.: Dorsal root entry zone lesions for pain relief. J. Neurosurg., 51:59, 1979.

[19] Kidd, D. H., Campbell, J. N., and Long, D. M.: Dorsal root ganglionectomy for failed back surgery syndrome: A 5-year follow-up study. J. Neurosurg., 74:236, 1991.

[20] North, R. B., Kidd, D. H., Zahurak, M., James, C. S., and Long, D. M.: Spinal cord stimulation for chronic, intractable pain: Experience over two decades. Neurosurgery, 32:384, 1993.

[21] Schvarcz, J. R.: Stereotactic high cervical extralemniscalmyelotomy for pelvic cancer pain. ActaNeurchir. (Suppl.), 33:431, 1984.

[22] Scoville, W. B.: Extradural spinal sensory rhizotomy. J. Neurosurg., 25:94, 1966.
[23] C. N.: Percutaneous radiofrequency denervation of spinal facets. J. Neurosurg., 43:448, 1975.

[24] Shealy, C. N., Mortimer, J. T., and Reswick, J.: Electrical inhibition of pain by stimulation of the dorsal column: Preliminary clinical reports. Anesth. Analg., 46:489, 1967.

[25] Shieff, C., and Nashold, B. S., Jr.: Stereotactic mesencephalotomy. Neurosurg. Clin. North Am., 1:825, 1990.

[26] M., Fischer, G., and Mansuy, L.: Posterior spinal rhizotomy and selective posterior rhizidiotomy. Progr. Neurol. Surg., 7:201, 1976.

[27] Sindou, M., Jeanmonod, D., and Mertens, P.: Ablative neurosurgical procedures for the treatment of chronic pain. Neurophysiol. Clin., 20:399, 1990.

[28] Snyder, S. H.: Opiate receptors and internal opiates. Sci. Am., 236:44, 1977.

[29] Spiegel, E. A., and Wycis, H. T.: Mesencephalotomy in treatment of intractable facial pain. Arch. Neurol. Psychiatry, 69:1, 1953.

[30] Spiller, W. G., and Martin, E.: The treatment of persistent pain of organic origin in the lower part of the body by division of the anterolateral column of the spinal cord. JAMA, $58: 1489,1912$

[31] Sweet, W. H.: Complications of percutaneous rhizotomy and microvascular decompression operations for facial pain. In Schmidek, H. H., and Sweet, W. H. (Eds.): Operative Neurosurgical Techniques. New York, Grune\& Stratton, 1988, p. 1139.

[32] Tasker, R. R.: Stereotactic surgery. In Wall, P. D., and Melzak, R. (Eds.): Textbook of Pain. London, Churchill Livingstone, 1994, p. 1147.

[33] Tasker, R. R., Dostrovsky, J. O., and Dolan, E. J.: Computerized tomography (CT) is just as accurate as ventriculography for functional stereotactic thalamotomy. Stereotact. Funct. Neurosurg., 57:157, 1993.

[34] vanKleef, M., Spaans, F., Dingemans, A., Barendse, G. A. M., Floor, E., and Sluijter, M. E.: Effects and side effects of a percutaneous thermal lesion of the dorsal root ganglion in patients with cervical pain syndrome. Pain, 52:49, 1993.

[35] J. K., Nauss, L. A., and Thomas, J. E.: Pain relief by intrathecally applied morphine in man. Anesthesiology, 50:149, 1979.

[36] H. T., and Spiegel, E. A.: Long-range results in the treatment of intractable pain by stereotaxic midbrain surgery. J. Neurosurg., 19:101, 1962.

[37] Yaksh, L., and Rudy, T. A.: Analgesia mediated by a direct spinal action of narcotics. Science, 192:1357, 1976.

[38] Young, J. N., and Wilkins, R. H.: Partial sensory rhizotomy at the pons for trigeminal neuralgia. J. Neurosurg., 79:680, 1993.

[39] Chambi, V. I.: Pain relief by electrical stimulation of the periaqueductal and periventricular gray matter. J. Neurosurg., 66:364, 1987. 